\title{
Comparison of Vertical Breast Displacement during Rope Skipping, Walking, Jogging and Running
}

\author{
X Sheng ${ }^{1}$, X Chen ${ }^{1,2,3^{*}}, \mathrm{Y} \mathrm{Li}^{1,2,3}, \mathrm{G} \operatorname{Sun}^{1,2,3}, \mathrm{H} \mathrm{Xie}^{1,2,3}$ \\ ${ }^{1}$ School of Textiles and Fashion, Shanghai University of Engineering Science, Shanghai, 201620, P.R. China \\ ${ }^{2}$ Textile Industrial Key Laboratory of Ergonomics and Functional Clothing, Shanghai University of Engineering Science, Shanghai, \\ 201620, P.R. China \\ ${ }^{3}$ Sino-British Joint Lab for Smart Sportswear, Shanghai University of Engineering Science, P.R. China
}

\begin{abstract}
In order to investigate the effect of exercise modality and bra type on vertical breast displacement, seven participants with similar ages and body types were selected to take part in this study. Three-dimensional motion capture system (NDI Optotrak Certus) was used to collect the data of vertical breast displacement when participants were walking at the speed of $5 \mathrm{~km} / \mathrm{h}$, jogging at the speed of $7.5 \mathrm{~km} / \mathrm{h}$, running at the speed of $10 \mathrm{~km} / \mathrm{h}$ on a treadmill, and rope skipping at the frequency of twice per second. It was found that there was no significant difference in vertical breast displacement between rope skipping, jogging and running. And the vertical breast displacement when wearing sports bra is significantly less than everyday bra in four exercise modalities. The results of this study might be useful for designing special sports bra for rope skipping modality.
\end{abstract}

\section{Introduction}

As a form of moderate-to-high intensity aerobic exercise, $[1,2]$ rope skipping is an effective way to lose weight, which is accepted and used by the majority of people.[3-5] Besides, previous researches showed that rope skipping exercise made a positive effect on vascular compliance, cardiopulmonary function, oxidative stress and antioxidant capacity.[5] Meanwhile, because of its low cost, rope skipping could be promoted to people from lower socioeconomic classes. Also, rope skipping is an activity which does not require a lot of space, hence is suitable for densely populated environments like school.[1] However, rope skipping includes a jumping component, which may cause excessive breast displacement. It is acknowledged that excessive breast displacement is related to breast discomfort even pain, which troubles many women, especially women with large breasts. $[6,7]$ Fortunately, breast displacement could be reduced by wearing sports bras.[8-10] Previous researches have investigated breast biomechanics during a range of exercise modalities, including treadmill walking[11], running[12] and swimming,[13] and found that increases in breast support (wearing bra) caused decreases in breast displacement.[13] But up to now, much less exploration on the vertical breast displacement during rope skipping was found.

Therefore, the first aim of this study was to assess vertical breast displacement during rope skipping, walking, jogging and running. The second aim of this study was to evaluate the effect of breast support on vertical breast displacement. Firstly, it was hypothesized that there would be significant differences in vertical breast displacement during rope skipping, walking, jogging and running. Secondly, it was hypothesized that breast motion would be significantly reduced as breast support increased from everyday bra to sports bra.

\section{Methods}

\subsection{Participants}

Seven healthy active women (mean \pm SD (Standard Deviation): age $=20 \pm 1 \mathrm{yr}$; breast circumference $=82 \pm$ $8 \mathrm{~cm}$; upper-breast circumference $=86 \pm 3 \mathrm{~cm}$; underbreast circumference $=73 \pm 2 \mathrm{~cm}$ ) who had no history of pregnancy, breast cancer or surgery were recruited for this study. According to the professional bra fitting criteria, each participant was fitted by an experienced bra fit assessor.[14] Four of participants were cup A (breast circumference minus under-breast circumference was 12$14 \mathrm{~cm}$ ), four of them were cup B (breast circumference minus under-breast circumference was $14-16 \mathrm{~cm}$ ), two of them were cup $\mathrm{C}$ (breast circumference minus underbreast circumference was $16-18 \mathrm{~cm}$ ), and one of them was cup D (breast circumference minus under-breast circumference was $18-20 \mathrm{~cm}$ )

\subsection{Experimental bras}

Everyday bra and sports bra used in the experiment were both common bras. The experimental sports bra used in this study was one of the basic styles of sports bra (NIKE ${ }^{\mathrm{TM}}$, China) (Figure 1), which was designed for

* Corresponding author: chenxn@sues.edu.cn 
medium intensity sports. Two different bra sizes ( $\mathrm{M}$ and $\mathrm{L}$ ) of this style were used. The fabric of the experimental sports bra is made of $88 \%$ recycled polyester and $12 \%$ spandex blended. The mesh fabric is made of $80 \%$ polyester and $20 \%$ spandex. Both the front side and back side of the liner are made of $100 \%$ polyester. Everyday bra used in this study was a common style (COSMO LADY, China), seen in Figure 2. The cup lining was made of $65 \%$ polyester and $35 \%$ cotton. The side lining was made of $84 \%$ polyamide and $16 \%$ spandex.

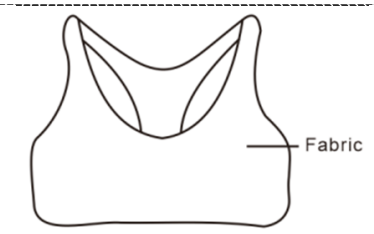

(a) Front side

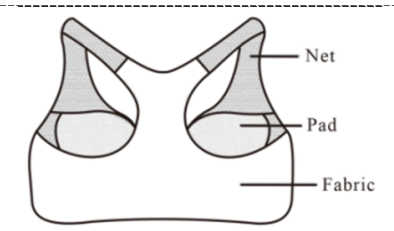

(b) Back side
Figure 1. Structure of sports bra, the front side (a) and the back side (b).

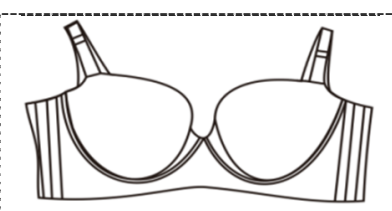

(a) Front side

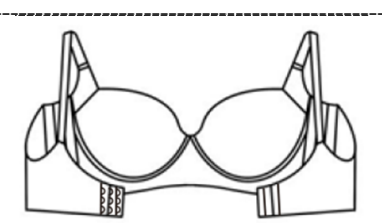

(b) Back side
Figure 2. Structure of everyday bra, the front side (a) and the back side (b).

\subsection{Measurement process}

This experiment was conducted in the artificial climate chamber of the human motion and ergonomics laboratory of the institute. Three-dimensional motion capture systems (NDI Optotrak Certus) with three infrared cameras were employed to collect the motion of the markers pasted on body and bra. The whole testing process included the following steps: warm-up, markers pasting and data collection. The laboratory coordinate system was established with the $\mathrm{X}$-axis positive rearward, Y-axis positive to the right, and Z-axis positive upward.

2.3.1 Warm-up. In order to prevent experiment accidents, all participants were provided with familiarization trials on the laboratory treadmill. During the familiarization period, they were allowed sufficient practice at a selfselected gait speed.

2.3.2 Markers Pasting. After warming up, participants were asked to put on the experimental bra. To quantify breast motion relative to trunk motion, one marker presenting the trunk movement was fixed on the suprasternal notch, another marker was positioned at left breast nipple to capture breast motion.

2.3.3 Data Collection. All data were collected for each participant, with participants required to complete four exercise trials in each bra condition. First, participants walked at the speed of $5 \mathrm{~km} / \mathrm{h}$ on the treadmill in the everyday bra condition. Data was collected $30 \mathrm{~s}$ after the start of exercise to ensure steady-state breast motion had been achieved. The data collection duration was $60 \mathrm{~s}$. Then, the same procedures were used in collecting breast motion data during treadmill jogging $(7.5 \mathrm{~km} / \mathrm{h})$, treadmill running $(10 \mathrm{~km} / \mathrm{h})$ and rope skipping (twice per second). Participants were given a sufficient rest period between trials. After all data collection in the everyday bra condition was completed, data in the sports bra condition was collected in the same procedures.

\subsection{Statistical analysis}

Assuming that the breast is symmetrical, the left breast was selected as the research object. That is, the data used in this study were all left breast data. Vertical breast displacement was the difference between the vertical coordinates of left breast nipple and suprasternal notch. The mean and SD values were calculated for each of the four exercise modalities for the average vertical breast displacement in the two bra conditions (everyday bra and sports bra).

In this study, all data of vertical breast displacement were checked for normality using a Kolmogorov-Smirnov test. A two-way ANOVA design with two within factors (exercise modality and bra type) was then conducted to reveal whether there was a significant effect of exercise modality and bra type on vertical breast displacement or interactions of exercise modality (walking, jogging, running, rope skipping) or bra type (everyday bra, sports bra). Bonferroni post hoc analyses were used to find where any significant difference exists. Statistical analyses were conducted in the Statistical Package for the Social Sciences (Version 25.0; SPSS Inc., Chicago, IL). The significance level was set at 0.05 .

\section{Results}

\subsection{Effect of exercise modality on vertical breast displacement}

Mean and SD of vertical breast displacement was calculated for each of four exercise modalities in the two bra conditions (Figure 3). During the trials, vertical breast displacement ranged from $3.957 \mathrm{~mm}$ to $13.360 \mathrm{~mm}$ whilst walking at the speed of $5 \mathrm{~km} / \mathrm{h}$, and ranged from $6.579 \mathrm{~mm}$ to $35.143 \mathrm{~mm}$ whilst jogging at a speed of $7.5 \mathrm{~km} / \mathrm{h}$. It was showed in Figure 3 that there was a dramatically increase of vertical breast displacement from walking to jogging. However, only slight changes were found between jogging, running and rope skipping. Meanwhile, the results of twoway ANOVA revealed that there was a significant effect of exercise modality on vertical breast displacement $(\mathrm{p}<$ $0.05)$. The statistic results showed that there was no significant exercise modality-bra type interaction $(\mathrm{p}=$ 0.403). Bonferroni post-hoc analyses determined that there were significant differences in vertical breast displacement between walking and running, walking and jogging, walking and rope skipping $(\mathrm{p}<0.05)$. 
Nevertheless, there was no significant difference in vertical breast displacement between running, jogging and rope skipping.

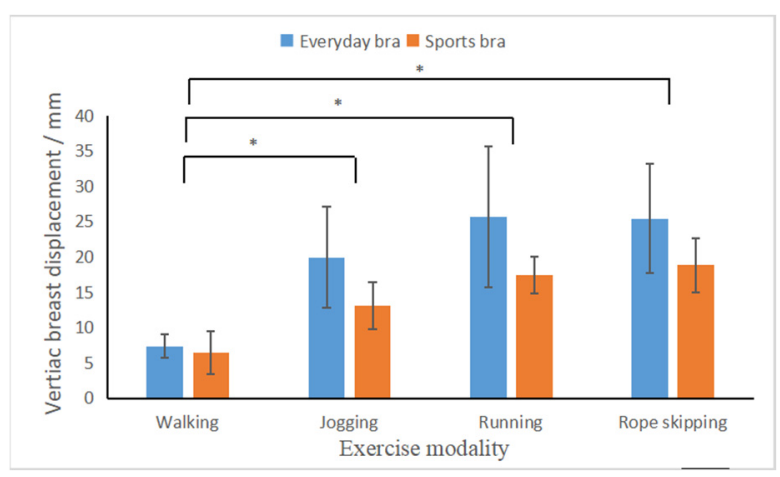

Figure 3. Mean $( \pm \mathrm{SD})$ vertical breast displacement at four exercise modalities in each breast support condition $(n=7)$.

* Significant difference in vertical breast displacement during exercise modalities $(\mathrm{p}<0.05)$.

\subsection{Effect of bra type on vertical breast displacement}

During the trials, vertical breast displacement ranged from $7.359 \mathrm{~mm}$ to $25.702 \mathrm{~mm}$ whilst the participants wearing everyday bra, and ranged from $6.490 \mathrm{~mm}$ to $18.832 \mathrm{~mm}$ whilst the participants wearing sports bra. The results of two-way ANOVA revealed that there was a significant effect of bra type on vertical breast displacement under each exercise modality $(p<0.05)$, whereby the vertical breast displacement in the everyday bra condition was significantly greater than when wearing the sports bra.

\section{Discussion}

This study investigated the effect of exercise modality and bra type on vertical breast displacement. It was found that there were significant differences between walking and running, walking and jogging, walking and rope skipping. However, no significant difference was found between rope skipping and jogging, rope skipping and running. It was also found that there was a significant reduction on vertical breast displacement whilst wearing a sports bra, compared to wearing an everyday bra. The details of these findings are as below.

\subsection{Effect of exercise modality on vertical breast displacement}

The first hypothesis could be partially accepted as the results of this study showed that vertical breast displacement differed significantly between walking and other three exercise modalities, confirming findings by Scurr et al.'s and Mason et al.'s research.[15-17] However, no significant difference was found in vertical breast displacement between rope skipping, jogging and running. These findings might be explained by the different intensity of these four modalities. Walking is a lowintensity exercise while running, jogging and rope skipping are all high-intensity exercises. The trunk movements of participants in running, jogging and rope skipping are similar in the vertical direction, with obvious up and down trunk movement. And it was speculated that such trunk movements would drive the breasts to do similar up and down movements. The findings suggested that breast motion research that analyzes treadmill jogging and running may be applicable to rope skipping, which might help to inform the requirements of breast support garments as a means to reduce breast pain for rope skipping.

\subsection{Effect of bra type on vertical breast displacement}

The results of this study confirmed that the vertical breast displacement in the sports bra condition was significantly reduced than when wearing the everyday bra, whatever the exercises modalities. Namely, sports bra is more effective in reducing vertical breast displacement than everyday bra. This corresponded with previous research $[18,19]$ and supported the second hypothesis of this study. According to Lu et al.'s research, the superiority of sports bra could be attributed to the tension caused by stretched bra fabrics and the compressed cup pad.[10] During exercise, the breast kinetic energy converts to the tensile energy of the fabric and pad material, and the compression energy of the pad material.[10] This may explain how vertical breast displacement was reduced in the sports bra condition. The findings of this study also supported the notion that women should be encouraged to wear sports bra during exercise to reduce the breast displacement and relieve the discomfort caused by excessive displacement. $[7,17,20]$

\subsection{Strengths and limitations}

A limitation of this study was that we only compared the vertical displacement between different exercise modalities. Previous studies had established that velocity and acceleration of movement during exercise and ground reaction forces that result from different breast support conditions were also factors affecting breast discomfort.[21-23] Therefore, future research might look to the comparisons of these three factors between rope skipping and other exercise modalities in different breast support conditions. There was also a limitation in the data processing. The marker representing breast motion was placed on the surface of bra instead of on the breast. The results might be biased because of possible relative displacement between bra and breast caused by rubbing during exercise. Furthermore, it was also acknowledged that the present study was limited in the small number of participants. Consequently, the results require further verification with more participants in the future.

Despite these limitations, to our knowledge, this study is the first to investigate the vertical breast displacement during rope skipping, and to compare the results during rope skipping with that during walking, jogging and running. Therefore, this information is important for designing special sports bras for rope skipping, which is expected to reduce excessive breast displacement and bra discomfort. 


\section{Conclusion}

This study found that there was no significant difference in vertical breast displacement between rope skipping and jogging, and between rope skipping and running. Therefore, findings in previous literature in breast motion during treadmill jogging $(7.5 \mathrm{~km} / \mathrm{h})$ or running $(10 \mathrm{~km} / \mathrm{h})$ could be applicable to rope skipping. Future research should consider comparing the velocity and acceleration of movement and ground reaction forces exerted on breast between rope skipping and other exercise modalities in different breast support conditions.

\section{Acknowledgement}

This study was funded by the National Natural Science Foundation of China (Grant No.11802171), the open fund of Key Laboratory of Clothing Design and Technology (KLCDT2020-01), Teacher Training Programs on Human Factor and Ergonomics (201901024017) and National Key Research and Development Program of China (2018YFC2000905).

\section{References}

1. Ha A S, Lonsdale C, Ng J Y Y and Lubans D R 2014 BMC Public Health 14535

2. Ridley K and Olds T S 2008 Medicine and science in sports and exercise 40 1439-46

3. Kang J, Zhou Y and He X 2008 China Sport Science and Technology 99-102

4. Hao M, Han W and Yamauchi T 2019 Asia-Pacific journal of public health 31 348-58

5. Zhang Y 2014 The effects of rope skipping on cardiopulmonary function in adolescents: an experimental study (Chengdu: Chengdu sport University)

6. Jie Z, Winnie Y and Ng S P 2011 Textile Research Journal 81 1234-48

7. Lorentzen, Deana, Lawson and LaJean 1987 Physician \& Sportsmedicine

8. Steve H and Joanna Scurr 2011 Sports Engineering 14

9. Maha S A A H M D and Frcsi 2000 The Breast Journal 6

10. Lu M, Qiu J, Wang G and Dai X 2016 Materials Today Communications 6 28-36

11. McGhee D E, Steele J R, Zealey W J and Takacs G J 2013 Appl Ergon 44 112-8

12. Steve H, Alex M and Joanna Scurr 2013 Proceedings of the Institution of Mechanical Engineers, Part P: Journal of Sports Engineering and Technology 227

13. Mills, Lomax, Ayres and Scurr 2015 Journal of Sports Sciences 33

14. McGhee D and J. Steele 2010 Journal of Science and Medicine in Sport $\mathbf{1 3}$

15. Scurr J C, Hedger J L W and Wendy 2011 Journal of
Sports ences 29 55-61

16. Scurr J, White J and Hedger W 2009 Journal of Applied Biomechanics 25 322-9

17. Mason B R, Page K A and Fallon K 1999 Journal of Science \& Medicine in Sport 2 134-44

18. Zhou J, Yu W, Ng Z and Hale J 2009 Journal of Fiber Bioengineering and Informatics 2 108-13

19. Bowles K A, Steele J R and Munro B 2008 Br J Sports Med 42 670-3

20. White J, Scurr J and Hedger W 2011 J Appl Biomech 27 47-53

21. White J L, Scurr J C and Smith N A 2009 Ergonomics 52 492-8

22. Deirdre E M 2007 British journal of sports medicine 12

23. Scurr J C, Hedger J L W and Wendy 2010 Journal of Sports ences 28 1103-9 